\title{
Experimental Tests and Performance Evaluation of a VHF Data Transceiver Prototype for Operation in the Antarctic Regions
}

\author{
Manuel PRIETO ${ }^{1}$, Miguel Angel DE PABLO ${ }^{2}$, Miguel RAMOS ${ }^{3}$, Juan Javier JIMENEZ ${ }^{3}$ \\ ${ }^{1}$ Dept. of Automática, University of Alcalá, Alcalá de Henares, Spain \\ ${ }^{2}$ Dept. of Geología, Geografía y Medio Ambiente, University of Alcalá, Alcalá de Henares, Spain \\ 3 Dept. of Física y Matemáticas, University of Alcalá, Alcalá de Henares, Spain \\ manuel.prieto@uah.es, miguelangel.depablo@uah.es
}

Submitted September 25, 2019 / Accepted January 15, 2020

\begin{abstract}
This paper presents the study and implementation results of a point to point radio data link carried out by the PERMASNOW project Research Team in the Gabriel de Castilla $(G d C)$ Spanish Antarctic Station (Deception Island, South Shetland archipelago, Antarctica) under challenging Non Line-of Sight (NLOS) conditions. Our final goal is to succeed in the remote data access of the multiple and dispersed measurement stations deployed in the surrounding area of the Antarctic Stations without the use of costly satellite communication systems. For so, a wireless sensor network scheme is proposed in which the key element is the node radio data transceiver characterized in this paper. The main design driver is the harsh Antarctic environmental conditions, which leads to a low power and rugged wireless solution. This study confirms the usefulness of the amateurradio bands and equipment, which mainly give versatility in frequencies, modulations and power configurations. The terrain topography shows to be the key factor in the short-range segment, notably affecting the propagation conditions. For the long-range segment, the best solution still shows to be a satellite link but promising ionospheric data link has been successfully tested.
\end{abstract}

\section{Keywords}

Data-link, sensor network, APRS, Antarctica, NLOS

\section{Introduction}

Antarctica is a unique place in the world for many reasons. For scientists, Antarctica is like a valuable laboratory due to its special environmental conditions, which have preserved the frozen continent of direct contamination by human action. Almost all science disciplines find their place in the Antarctica: Biology, Geophysics, Geology, Oceanography, Botanic, even Space and Astronomy. The University of Alcala (Spain) (UAH) have been present in Antarctica for more than 20 years analyzing the permafrost and the active layer evolution in the frame of the climate change study [1-3]. The UAH Permafrost Research Team counts on 84 measurement sites distributed along Deception and Livingston islands, both in the South Shetland Archipelago. One of the main parameters used to characterize the changes in the permafrost active layer is the ground temperature. For so, a set of temperature sensors are placed at different depths in the ground inside several isolated boreholes. The deepest borehole is at Livingston Island, which goes up to 25 meters deep in the frozen ground. By the temperature measurement, it can be determined the active layer depth that is the soil surface layer formed when the permafrost thaws and is frozen again. The basic station is typically complemented with soil surface and air temperature sensors as well as temperature sensors in a wood steak to approach the snow cover thickness (Fig. 1).

The monitoring stations are operative all over the year, taking measurements and logging these data locally until the research team can retrieve them during the annual Antarctic fieldtrips in the Antarctic summer period. Antarctic campaigns are short and very conditioned by the meteorology. In this sense, time is gold in Antarctica. Considering that the 84 measurements sites result in more than 500 sensors, the researcher dedicates most of the time in Antarctica to collect data and perform maintenance activities. Figure 2 shows the traditional method for data collection.

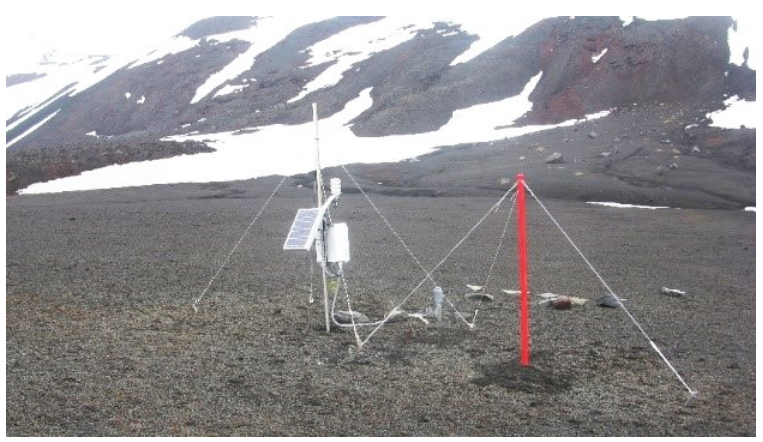

Fig. 1. DECBOR3 monitoring site in Deception Island. 


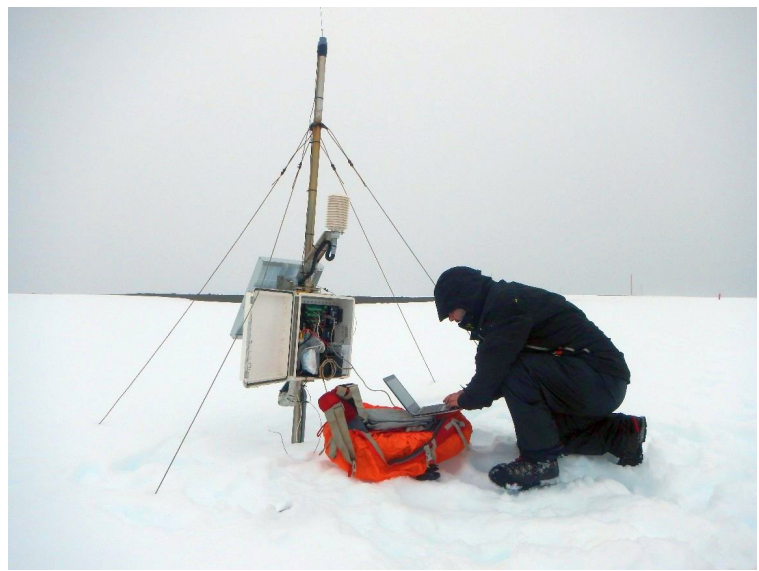

Fig. 2. Manual collecting data procedure.

The main objective of the study presented hereby is to have a reliable data communication solution that allow us to remotely have access to the data, so the valuable time in Antarctica can be dedicated to explore new areas and perform new research activities. Radio links will help the researchers in several aspects. Before travelling to Antarctica, the status of the measurement sites could be known. This helps in the campaign planning, procuring in advance only those items that are known to be damaged and which require maintenance tasks. This also contributes to decrease the cost of the campaign as well as the amount of material to be transported to Antarctica. If for any reason, the campaign is cancelled or the researcher cannot travel to Antarctica, data are still available: the science of the year is not lost. Finally, once in Antarctica, it prevents the researcher to walk long distances or go into protected areas, to those measurement sites that are working properly.

The establishment of a radio data link is very common and easy to implement with the new available technologies. Nowadays, thanks to the boom of the Internet of Things (IoT), not only engineers but also hobbyists, or even students count on many electronic devices to create wireless networks easily. Development platforms such as Arduino, Raspberry, etc., provides low cost LoRa, Wi-Fi, Bluetooth or Zigbee modules to create radio data links all in one go. The challenge is not to establish a radio data link, but to make it work under the Antarctic environmental conditions for a complete Antarctic year, without any maintenance. In addition, it has to work in the best but also in the worst conditions of line of sight (LOS). The first thing that comes to someone's mind is that Antarctica can be a very cold place, so the electronics should withstand low temperatures such -30 or -40 degrees Celsius, or even less $\left(-80^{\circ} \mathrm{C}\right.$, in the inner sector of Antarctica). Nevertheless, temperature is not the main design driver, since the electronics are rarely exposed outside a box. The inner heat produced by the electronics, together with the appropriate isolation material, can create a comfortable environment for the electronics inside the box. In Antarctica, the main problems for radio-communication electronics are, with no doubt, power generation and wind, together with the fact that maintenance is very limited.

\section{Materials and Methods}

The proposed networking communication scheme is shown in Fig 3. It is divided into a short range segment and a long range segment. The short range refers to the radio links between sensors and the Gabriel de Castilla (GdC) Spanish Antarctic Station. The long-range segment is the connection between the GdC Antarctic Station and the final user in Spain.

The selected site taken for the pilot experience is named Crater Lake CALM-S (Circumpolar Active Layer Monitoring) (CL from here) located in the Crater Lake Plateau of Deception Island (62 59'08,04629"S, 6040’48,21453"W). As shown in Fig. 4 and Fig. 5, it is approximately located $1 \mathrm{~km}$ far from Gabriel de Castilla Antarctic Station (GdC) and it presents an irregular orography, so no line-of sight is present between CL and GdC. CL was specially selected for the experiment because it is considered a good representative of a complete permafrost measurement site.

DECBOR3 is a permafrost measurement station composed of one thermometric chain of 14 temperature sensors, plus air temperature and humidity sensor, connected to a Campbell Scientific CR1000 data logger. Power is provided by a SP-10 W Campbell Scientific solar panel (https://www.campbellsci.com). The power system is completed with an Enersys Genesis $26 \mathrm{Ah}$ battery and solar panel regulator. DECBOR3 is one out of the seven boreholes drilled in CL and is the central node of the permafrost measurement network (red point in Fig. 3).

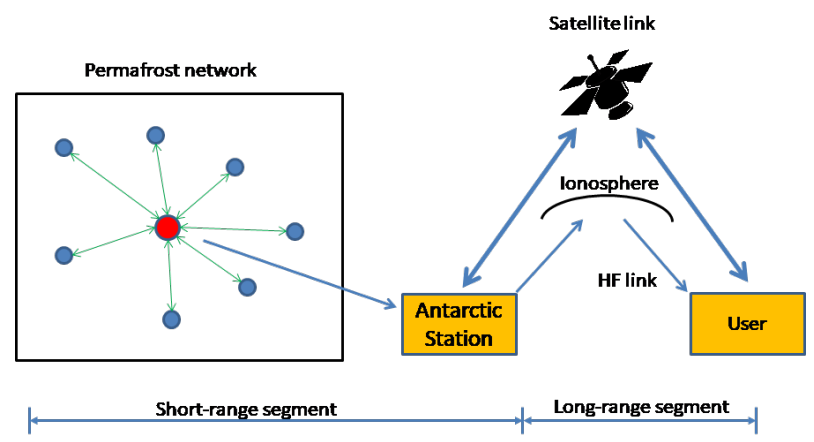

Fig. 3. Communication system block scheme

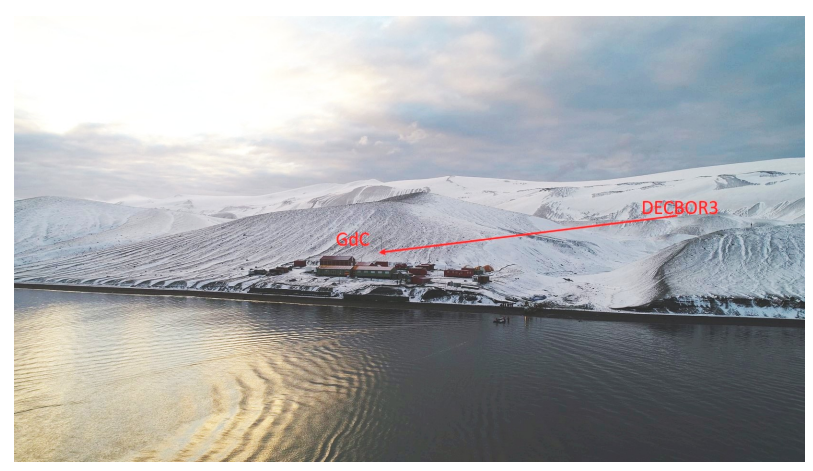

Fig. 4. GdC and DECBOR3 locations. 


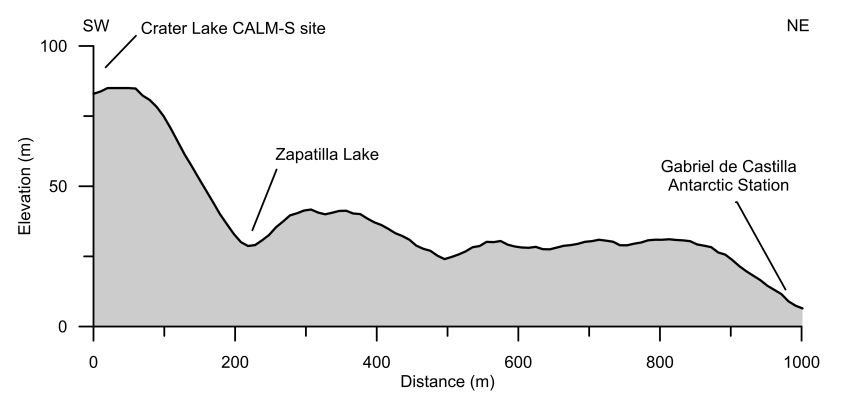

Fig. 5. Topographic profile of the experiment set-up.

Our final goal is that the rest of the nodes of the permafrost network send the data to DECBOR3, as the central node, and DECBOR3 retransmits the data to the Antarctic Station. This work is mainly focused in this specific section of the short segment data link, which showed to be the most problematic one in terms of signal attenuation.

The first thing to do is to perform a basic power budget to have an estimation of the RF power that can be used to establish the radio link. The analysis should be done always under the worst-case conditions. According to the Spanish Meteorological Agency (AEMET) [4] the worst-case solar radiation takes place in winter (June-July-August) with an average global radiation of $1674 \mathrm{~kJ} / \mathrm{m}^{2}\left(465 \mathrm{Wh} / \mathrm{m}^{2}\right)$. Solar panel features are usually given assuming $1000 \mathrm{Wh} / \mathrm{m}^{2}$ at $25^{\circ} \mathrm{C}$ (Standard Test Conditions). According to the manufacturer, SP10 solar panel has an effective area of $0.06 \mathrm{~m}^{2}$ approximately, which leads to an efficiency of around $16 \%$. In this situation, the solar panel can only provide a maximum of $4.65 \mathrm{Wh}$ at $25^{\circ} \mathrm{C}$ during winter. Fortunately, temperatures in winter are lower than $25^{\circ} \mathrm{C}$ in Antarctica, so no penalty in power output due to temperature is expected. Nevertheless, snow and frost deposition in the active area will decrease output power generation. Taking a recommended safety margin of $50 \%$, the maximum output power would be of $2.32 \mathrm{Wh}$. The question now is how far the radio signal can get with an output power of roughly $2 \mathrm{~W}$ and whether the radio link between DECBOR3 and GdC is feasible or not.

Operating frequency is highly important because propagation features, bandwidth, antenna size and path losses depend on this parameter. The selected bands for the shortrange segment are VHF and UHF. These bands do not require big antennas and sophisticated fixing elements, which is compatible with a field experiment in Antarctica due to the high wind gusts. In these bands, radio signals follow a line-ofsight propagation. Line-of-sight refers to radio waves, which travel directly in a line from the transmitting antenna to the receiving antenna, although this does not necessarily imply a cleared sight path. Radio waves can pass through obstructions more easily, as the frequency is lower. Path loss (PL) is directly related with the radio waves propagation. Like light waves, radio waves are also affected by the phenomena of reflection, scattering and diffraction, which impact in the propagation features of the radio link. Radio wave propagation is a constant subject of study, evolving as new technologies emerge [5]. In general, range determination involve five main parameters:

- Operating frequency.

- RF output power $\left(P_{\mathrm{TX}}\right)$ and receiver sensitivity.

- Transmitter and receiver line losses $\left(L L_{\mathrm{TX}}, L L_{\mathrm{RX}}\right)$.

- Transmitter and receiver antenna gain $\left(G_{\mathrm{AntTX}}\right.$, $G_{\text {AntRX). }}$.

- Path loss (PL).

Equation (1) relates the above parameters in the link budget calculation. The link margin is the difference between the power at reception (PRX) and the sensitivity. The link margin must be positive and, depending on the modulation, a minimum margin may be required.

$$
\begin{gathered}
P_{\mathrm{RX}}=P_{\mathrm{TX}}+G_{\mathrm{AntTX}}-L L_{\mathrm{TX}}-P L-L L_{\mathrm{RX}}+G_{\mathrm{AntRX}}, \\
F P L[\mathrm{~dB}]=32.44+20 \log D+20 \log f
\end{gathered}
$$

Even when in realistic radio communication the path is never free, the basic propagation equation is the Free-space Path Loss (FPL) (2) [6], which relates the signal loss with the distance in kilometers $(D)$ and the frequency in megahertz $(f)$. The higher the distance or the frequency, the higher the losses. For that reason, it is important to operate at low frequencies (VHF) instead of higher frequencies in the UHF band, such as those used by Wi-Fi or Bluetooth devices $(2.4 \mathrm{GHz})$. The design proposal relies on amateur-radio bands and equipment. Amateur-radio bands allow the user to use higher transmission power than the ISM (Industrial, Scientific and Medical) bands. The so-called Short Range Devices (SRD) mainly use ISM bands. Transmission power in SRD devices is typically of $25 \mathrm{~mW}(14 \mathrm{dBm})$. Therefore, achievable ranges with ISM devices are very short when there is not a cleared sight between transmitter and receiver. Effective range is lower than 100 meters in most applications. Longer ranges of around 20 kilometers can be achieved in specially clear line of sight scenarios and using high gain directive antennas [7] [8]. The main advantage of ISM band is that is license free, precisely because of the very limited maximum power emission established by international radio regulations. This limitation in power makes also possible the use of small batteries in field sensors. In turn, amateur-radio bands require the user to have an amateur-radio license but at no cost. Most universities and research institutes do have an amateur-radio license which can be used for this kind of experiments. In real life, the truth is that there are obstacles in the radio path that may strongly attenuate the radio signal. This is why we consider that SRDs operating at ISM bands are not good for difficult NLOS scenarios. More power and lower frequencies are needed, so VHF amateur-radio band is the option to use. For the pilot experience, the transmitter uses European APRS frequency at $144.800 \mathrm{MHz}$. APRS (Automatic Packet Reporting System) is a mature digital communication system also known as Radio-packet. It is based on AX.25 


\begin{tabular}{|c|c|c|c|c|c|c|c|c|}
\hline $\begin{array}{c}\text { Center } \\
\text { Frequency }\end{array}$ & $\begin{array}{c}\text { Transmit } \\
\text { Power }\end{array}$ & $\begin{array}{c}\text { Max. Distance } \\
\text { NLOS }\end{array}$ & $\begin{array}{c}\text { Max. Distance } \\
\text { LOS }\end{array}$ & $\begin{array}{c}\text { Receiver } \\
\text { Sensitivity }\end{array}$ & Bandwidth & Data Rate & Packet size & Protocol \\
\hline $144.8 \mathrm{MHz}$ & $2 \mathrm{~W}$ & $1.1 \mathrm{~km}$ & $24.4 \mathrm{~km}$ & $-95 \mathrm{dBm}$ & $8 \mathrm{kHz}$ & $1200 \mathrm{bps}$ & 128 bytes & $\mathrm{AX} .25$ \\
\hline
\end{tabular}

Tab. 1. Data link performance.

protocol and Bell 202 AFSK modulation [6] [9]. The widely used industrial HART standard is also based on Bell 202 modulation. The key thing of APRS is its simplicity and that it globally used all around the World by amateur-radio operators, aircrafts, cubesats and vessels. It allows an unlimited number of nodes. In addition, there exist thousands of IGATEs that transfer the APRS traffic into Internet. Data can be access in real time through specific servers such as Findu (http://aprs.fi). APRS is a connectionless oriented protocol, so transmitter just takes care of transmitting a packet. There is no handshaking between transmitter and receiver, reducing the traffic and saving power. In Antarctica, the main rule not to forget is to keep things as simple as possible (Tab. 1).

An STM8 microcontroller board (Fig. 6) interfaces the CR1000 datalogger with the RF transmitter, producing the APRS data frames and setting the scan slot times. This PC104 sized board may be equipped with Radiometrix HX1 FM transmitter or with the CC1000 FSK transceiver. Before its use in Antarctica, it has been widely tested in stratospheric balloon experiments. Stratospheric balloon is a good example of how long ranges can be achieved when there is a line of sight scenario. Successful radio links can be confirmed at high altitudes up to $28 \mathrm{~km}$ with just $300 \mathrm{~mW}$ of RF power, while on ground, coverage ranges goes down roughly 500-1000 meters. An ABS box contains the radio-modem made of the RF transceiver and the microcontroller card.

Antenna gain is also a key factor in determine the link budget. High gain antennas usually means big antennas, so careful must be taken when used in Antarctic conditions. The antenna to be used in the field experiments such as DECBOR3 should be light and flexible, which translates in a basic wire omnidirectional antenna with $0 \mathrm{~dB}$ of gain. Directive antennas provides gain, but are not a good option in general, since it is easier that strong wind can change the direction of the antenna, modifying the alignment between transmitter and receiver, making the signal fade. For the experiment, the transmitter antenna is a UT-108V antenna, from Nagoya. The receiver shall use the CHL-25S from Comet, with a maximum gain of $2.15 \mathrm{dBi}$.

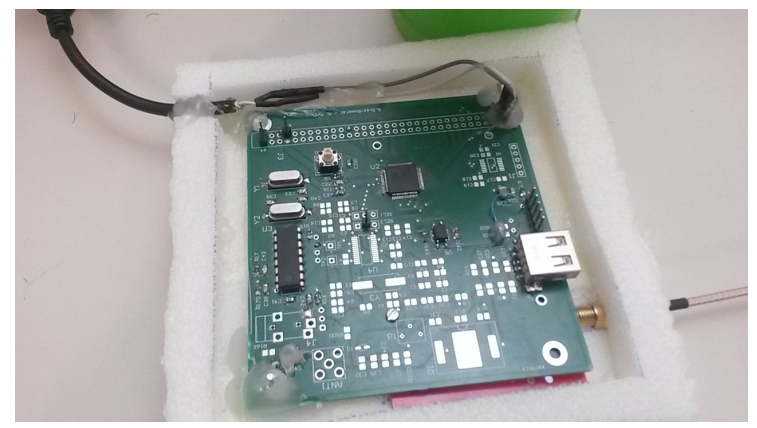

Fig. 6. Microcontroller card.
As for the GdC end-point, a Raspberry Pi computer module controls the SDR receiver. The receiver selected is an RTL-SDR v3 dongle (http://www.rtl-sdr.com). This is a Software Defined Radio (SDR) device with a wide frequency range from $500 \mathrm{kHz}$ to $1766 \mathrm{MHz}$ and with a maximum sensitivity of $-110 \mathrm{dBm}$. The Raspberry Pi tunes the receiver at the desired frequency, decodes APRS data frames and dialogues with the satellite link provided by the Antarctic Station. GdC also provides power to the receiver, which average power consumption is of $5 \mathrm{~W}$, obtained from a wind turbine during winter. For the long-range segment of the communications system, GdC uses Inmarsat platform as the satellite link service during winter season. Raspberry $\mathrm{Pi}$ is connected to the satellite datalogger via an Ethernet connection.

\section{Results and Discussion}

\subsection{Short Range Segment}

Before travelling to Antarctica, detailed link budget analyses were done with Radio Mobile software (http://www.ve2dbe.com/rmonline.html). Radio Mobile is a free software tool used to predict the performance of a radio system. It uses digital terrain elevation data for automatic extraction of path profile between an emitter and a receiver. It is based on the ITS Irregular Terrain Model (Longley-Rice) radio propagation model [10]. The analysis were completed with field radio coverage experiments performed at different locations in Alcalá de Henares and Guadalajara (Spain) to confirm the suitability of the solution to be installed in Deception Island. First experiment was performed with a Moteino modules (https://lowpowerlab.com). It is a small PCB with an Arduino and an RFM69 FSK or RFM95 LoRa transceiver operating at $433 \mathrm{MHz}$. As expected, the range was very short for our needs. No more than 25 meters with both transmitter and receiver indoors, and no more than 1000 meters outdoors with an open line of sight. ISM bands were discarded after this experiment for the link between DECBOR3 and GdC. LoRa or FSK transceivers operating in the ISM bands are ideal for the networking connection of the rest of the CL nodes to DECBOR3, where there is maximum distance of 200 meters with a clear line of sight and good weather conditions.

Next field experiments were done with HX1 transmitter $(300 \mathrm{~mW})(\mathrm{http} / / / \mathrm{www}$. radiometrix.com/) and Baofeng UV-3R (2000 mW) transceiver (https://baofengtech.com) tuned at $144.80 \mathrm{MHz}$ and APRS. Ranges of more than 3 kilometers were easily achieved in the busy urban area of Alcalá de Henares. This experiment confirmed 


\begin{tabular}{|c|c|c|c|c|c|c|c|c|c|}
\hline & PTX $[\mathrm{dBm}]$ & GAntRX & LLTX & PL & LLRX & GAntTX & PRX $[\mathrm{dBm}]$ & SRX & Margin \\
\hline Points A/B & 33.01 & 0 & 0 & 94.4 & 0 & 2.2 & -59.1 & -95 & 35.9 \\
\hline
\end{tabular}

Tab. 2. Minimum Link Margin (dB) with low resolution DEM.

HX1 transmitter as a good candidate for the first experience in Antarctica. Nevertheless, it was decided to go ahead with the solution based on Baofeng UV-3R to test the limits of power generation of DECBOR3 site. Baofeng UV-3R power consumption is of $5.5 \mathrm{~W}$ in transmission mode, which doubles more than the winter limit of $2.32 \mathrm{~W}$ calculated in Sec. 2 . The pilot experiment was finally installed in February 2017 and first results were analyzed one year later. It was detected an important gap of two months in the data, coinciding with the worst case illumination conditions. As expected, even with a $10 \mathrm{~W}$ solar panel, the average power consumption must be kept below $5 \mathrm{~W}$. In addition, data showed an important packet lost ratio of $50 \%$.

During campaign 2017-2018, short-range link tests were performed at different locations around the Antarctic Station (Fig. 7). The radio signal was received perfectly at the Scientific Module (point B) with no packet loss, but 30 meter far away (point A), the packet loss ratio increased notably. The location of the satellite modem forced us to place our receiver in point A. Several antennas and different receivers were tested, with the same results. The main difference between point A and point B in Fig. 7 is that point A has a significant obstruction in front due to JB Hill and the warehouse. In point $\mathrm{B}$, the radio signal is able to find its way through the Mekong Valley. The conclusion was that, even with $2 \mathrm{~W}$ of RF power, the radio system was not able to cover a distance of just 1 kilometer, due to losses added by obstacles. This finding was only possible after the field test. The radio-link model showed no problem at all, with an exceptional reception level and link margin according the parameters of (1) (Tab. 2). Transmitter and receiver line losses may be considered negligible meanwhile cable distances between antenna and receiver are kept short (below 3 meters). Its contribution is typically less than $1 \mathrm{~dB}$, as attenuation for the commonly used RG-58 cable is $0.16 \mathrm{~dB} / \mathrm{m}$ at $145 \mathrm{MHz}$. It is important to remark that the higher the frequency is, the higher the line loss is.

However, the experience on the field said the opposite. The radio link did not work. The reason behind is that the terrain model used in the first analysis was not accurate. Radio Mobile uses Digital Elevation Models (DEM) to calculate the obstruction losses. It was not until a high resolution DEM provided by the Spanish Polar Committee was used in the analysis that Radio Mobile spotted out the coverage problem with LOS conditions for GdC.

Due to power restrictions, it is not recommended to increase the transmitter power. Moreover, there is only a difference of $3 \mathrm{~dB}$ from using $2 \mathrm{~W}$ to $4 \mathrm{~W}$ of output power. The $4 \mathrm{~W}(+3 \mathrm{~dB})$ would not solve the problem and would be incompatible with the power generation system capabilities.
On the other hand, due to the strong wind gusts, it is not possible to use big antenna system or raise much the antennas. Move the transmitter or the receiver to a better location is not possible either. The only feasible solution is then to act over the receiver. Figure 8 shows the coverage map for the link parameters used in the link analysis by the use of Radio Mobile software using the high resolution DEM. All colors except blue and grey mean a successful link, and unfortunately, GdC is in the very limit. Table 3 shows the results of the radio link modeling with Radio Mobile for points A and B using the high resolution DEM. The sensitivity of the receiver (SRX) is $-95 \mathrm{dBm}$, which was experimentally obtained at laboratory. The theoretical and experiments results show how the minimum link margin for AFSK is of $20 \mathrm{~dB}$ and how a minimum difference of roughly $5 \mathrm{~dB}$ between point $\mathrm{A}$ and point $\mathrm{B}$ can make the difference between success and failure. These results were corroborated performing a set of measurements on different locations in Spain with similar terrain elevation profile as the one of DECBOR3.

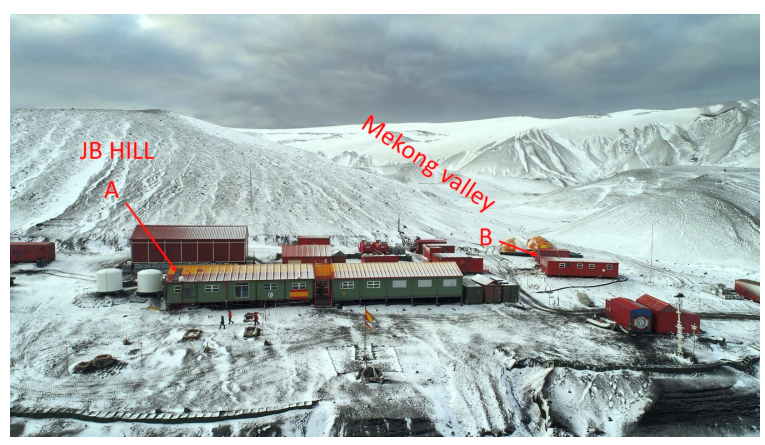

Fig. 7. Locations for the receiver in two different sites at the roofs of the GdC Station modules.

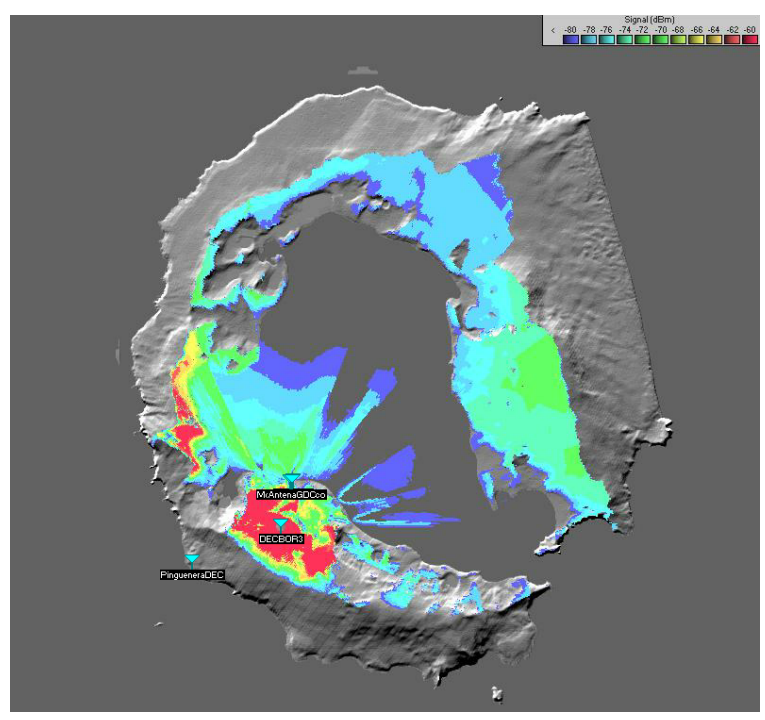

Fig. 8. Radio coverage map in Deception Island. 


\begin{tabular}{|l|c|c|c|c|c|c|c|c|c|}
\hline & PTX $[\mathrm{dBm}]$ & GAntRX & LLTX & PL & LLRX & GAntTX & PRX $[\mathrm{dBm}]$ & SRX & Margin \\
\hline Points A & 33.01 & 0 & 0 & 115.1 & 0 & 2.2 & -79.9 & -95 & 15.1 \\
\hline Points B & 33.01 & 0 & 0 & 110.1 & 0 & 2.2 & -74.8 & -95 & 20.2 \\
\hline
\end{tabular}

Tab. 3. Minimum Link Margin (dB) with high resolution DEM.

The solution was to use a low noise amplifier (LNA) just between the antenna and the receiver. A LNA adds gain to the receiver, but the most important contribution of an LNA is that it lowers down the noise figure (NF) of the receiver, increasing its sensitivity [7]. Noise factor (F) of a system is the defined as the ratio of signal-to-noise ratio (SNR) at the input with respect to the SNR at the output (3). Basically, the noise factor of an active element in the receiver, such as an amplifier with gain $G$, shows the amount of noise introduced by such element [11]. Ideally, a noise factor of 1 , would indicate that the active element does not introduce any noise $\left(N_{\mathrm{x}}=0\right)$. In the case of an amplifier, both the input signal and the input noise would be equally amplified, so the SNR would remain the same.

$$
\begin{gathered}
F=\frac{S N R_{\mathrm{i}}}{S N R_{\mathrm{o}}}=\frac{N_{\mathrm{x}}+G \times N_{\mathrm{i}}}{G \times N_{\mathrm{i}}}, \\
F=F_{\mathrm{LNA}}+\frac{F_{\mathrm{SDR}}-1}{G_{\mathrm{LNA}}}=1.25+\frac{5.01-1}{200}=1.27, \\
N F=10 \times \log (F)=10 \log (1.27) \approx 1.04 .
\end{gathered}
$$

In practice, the SNR at the output is always lower than at the input, since the amplifier introduces some extra noise $\left(N_{\mathrm{x}}\right)$. Considering a two active elements in the receiver chain, i.e., the LNA and the SDR receiver, the overall noise factor would be given by (4). The LNA4ALL device (Fig. 9) has been selected. It has a NF of $1 \mathrm{~dB}$ (1.25). Adding this LNA, the overall NF of the receiver chain is reduced roughly down to $1 \mathrm{~dB}$ eq. (5). With a typical $N F=7(5.01)$ of a receiver without LNA, the maximum improvement in the link margin would be of $6 \mathrm{~dB}$. Reducing the bandwidth and the NF imply higher sensitivity. It is important to remark that $8 \mathrm{~W}$ of RF power would be required in the transmitter to equal the gain of $6 \mathrm{~dB}$ given by the LNA. In addition, LNA4ALL device adds an maximum gain of $23 \mathrm{~dB}(\approx 200)$.

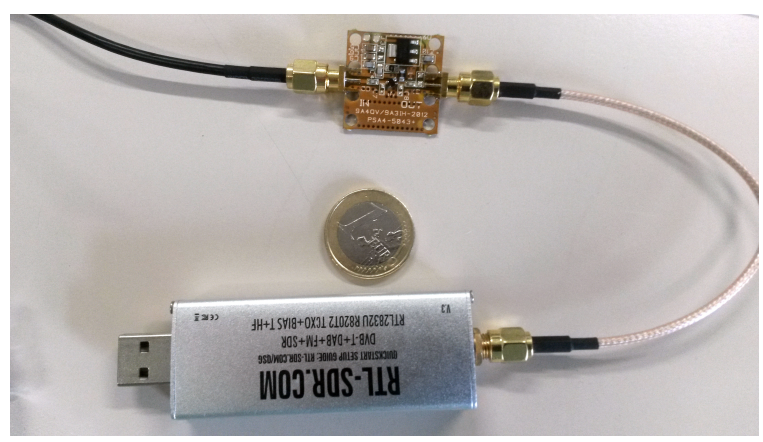

Fig. 9. LNA connected to the receiver.

\subsection{Long Range Segment}

As for the long-range segment, a satellite link is the most reliable way of sending data to any place in the World. Its main drawbacks are: 1) power consumption of the satellite modem and 2) the cost per bit. A satellite modem usually demands a peak current drain of $1 \mathrm{~A}$ or $2 \mathrm{~A}$. Despite this high current peak, it can be powered with the adequate solar panel and a battery. The main drawback is that the satellite is a costly solution. A commercial satellite link is not affordable in our case, where our research team has more than 84 measurement sites. It does not make sense either that each research team pays a satellite link. It would be wiser that the nearest Antarctic Station would provide this service. This would reduce costs. This is the reason why this experience makes use of the Inmarsat satellite link offered by the GdC Antarctic Station. Non-commercial satellites such as ARGOS-METOP, the ISS, GOES or even cubesats which have coverage over Deception Island have been explored as an alternative. Nevertheless, our experience during campaign 2015-16 tells us that the most reliable solution is a commercial satellite.

Nevertheless, in order to save costs, two alternative experiments were carried out. The first experiment consisted in establishing a radio link with two LEO satellites: The International Space Station (ISS) and the SERPENS cubesat [12]. The rationale behind is that LEO satellites may be a good alternative to traditional commercial Iridium or Inmarsat satellites, since LEO satellites may offer a long-range data communication system at no cost. Any amateur-radio operator can access the ARISS APRS digipeater on board ISS [13] very easily with a basic transceiver and an antenna. ISS is flying at an altitude of about 400 kilometers and its orbit has an inclination of about 51.6 degrees. Its footprint covers South Shetland Islands in Antarctica, so ISS was considered a good option to have a free satellite data link. SERPENS was a cubesat directly released from the ISS, so it had the same orbit and ground track approximately. Both experiments showed that the establishment of a radio data link requires a directive antenna and a minimum of $25 \mathrm{~W}$ output power. In addition, the directive antenna pointing must be periodically corrected according to the orbital TLE (Two Line Element) evolution; frequency Doppler Effect must be also considered; and satellite access is limited to one or two passes a day of 5 to 10 minutes in the best case. In conclusion, LEO satellites are discarded for this kind of applications, since maintenance is not possible during winter season and power supply is very limited. In addition, LEO satellites footprint only covers outer part of Antarctica, not suitable for latitudes beyond 62-64 degrees. 
The second long-range experiment tried to assess the feasibility of establishing a radio data link via ionospheric propagation. The experiment consisted in a FT-847 amateurradio transceiver operating at 20 -meter band $(14 \mathrm{MHz})$ and 40-meter band (7 MHz). A G5RV dipole antenna was used. We succeeded in establishing contact with Spanish and South America radio stations, but at a cost of using $100 \mathrm{~W}$ of RF output power. In addition, the propagation conditions strongly affected the radio signal quality. We drew two main conclusions: 1) HF data link is possible and 2) since the power should be lower down to 5 or $10 \mathrm{~W}$, the distance expectations should be limited to reach South America (1000 or $2000 \mathrm{~km}$ maximum). As an alternative to satellites, ionospheric link using low power modes, such as FT8, should be explored as a future solution. This type of digital modes in HF works at very low rate, being capable of reaching long distances with a radiation power of just $10 \mathrm{~W}$.

\section{Conclusions}

Something that may seem trivial, such as powering and establishing a short radio data link, can become a difficult task in Antarctica. This work is precisely the result of these difficulties. The experience drawn along three Antarctic campaigns shows how theory is one thing and reality is another, what in Antarctica may notably differ.

The main objective of having remote access to the data produced by our experiments in Antarctica for a full Antarctic year is achieved. In terms of power, this work has set a maximum limit of $2 \mathrm{~W}$ for the average power consumption of the electronics, considering the typical configuration made of a $10 \mathrm{~W}$ solar panel and a battery of $26 \mathrm{Ah}$. In higher latitudes south, this limit may decrease, conditioned by the number of hours of light in the wintertime. This work confirms that power should be kept as minimum as possible and there must be taken safety margins in the design. In this sense, the maximum RF power is limited as well, with a maximum of $2 \mathrm{~W}$.

The achievable range with $2 \mathrm{~W}$ may vary from roughly 1 kilometer to more than 30 kilometers, depending on the orography of the terrain and the Line of Sight conditions. It is important then to have a detailed model of the terrain to help in the radio link analysis (theory). Models are very useful in the early phases of the design. However, this does not guarantee that the link will work, even less in Antarctica. All models have limitations and accuracy is limited. Different models can give different answers. It would be helpful to have real coverage maps extracted from empirical measurements (reality).

RF link margin is important. It has been determined that the link margin should be above $20 \mathrm{~dB}$. In the case of having a smaller link margin, it is recommended first to act over the non-consuming elements such as antennas (raising the antenna for instance), or by lowering the frequency and the data rate. If finally, additional active elements must be included, then it is recommended to focus the efforts on increasing the sensitivity of the receiver. An LNA may be a good solution as demonstrated.

Unless ISM devices are allowed to offer higher output power or use lower frequencies such as those in the VHF band, amateur radio bands are a good option to solve the problem in scenarios where radio signal quality is highly affected by NLOS conditions. Amateur-radio offers all kind of open source modulation techniques, cheap equipment and electronic devices and a huge community of people interested in radio-communication.

\section{Acknowledgments}

The authors want to thank the entire army crews of the Gabriel de Castilla Spanish Antarctic Station for their help and support. Our special thanks to the Spanish Polar Program, the Unit of Marine Technology from the Spanish National Research Council, as well as to the Hespérides Research Vessel crews (from Spanish navy) involved on Antarctic Campaigns for making this work possible. Thanks for the interest of Observatorio de Rayos Cósmicos Antártico project (reference CTM2016-77325-c2-1-P). Finally, our gratitude to Mr. Roger Coudé VE2DBE for helping us to export the high resolution DEM of Deception Island to the Radio Mobile tool 73!. This research was funded by Ministerio de Economía y Competitividad (Spain), grant number CTM2014-52021-R.

\section{References}

[1] RAMOS, M., DE PABLO, M.A., MOLINA, A., et al. Recent shallowing of the thaw depth at Crater Lake, Deception Island, Antarctica (2006-2014). Catena, 2017, vol. 149, no. 2, p. 519-528. DOI: 10.1016/j.catena.2016.07.019

[2] RAMOS, M. Automatic device to measure the active permafrost layer near the Spanish Antarctic Station. Terra Antarctica, 1995, vol. 3, no. 1 , p. 61-63.

[3] DE PABlo, M.A., RAMOS, M., MOLINA, A., et al. Thaw depth spatial and temporal variability at the Limnopolar Lake CALM-S site, Byers Peninsula, Livingston Island, Antarctica. Science of the Total Environment, 2018, vol. 615, p. 814-827. DOI: 10.1016/j.scitotenv.2017.09.284

[4] GARCIA, M. B., LOPEZ, F. V. AEMET en la Antártida Climatología y Meteorología Sinóptica en las Estaciones Meteorológicas Españolas en la Antártida. (AEMET in the Antarctica. Synoptic Climatology and Meteorology in the Spanish Weather Stations in the Antarctica. Ministry of Agriculture, Feeding and Environment). (in Spanish) Ministerio de Agricultura, Alimentación y Medio Ambiente, 2015. ISBN: 9788478370931

[5] SARKAR, T. K., JI, Z., KIM, K., et al. Survey of various propagation models for mobile communications. IEEE Antennas and Propagation Magazine, 2003, vol. 45, no. 3, p. 51-82. DOI: 10.1109/MAP.2003.1232163

[6] ARRL Inc. The ARRL Handbook for Radio Communications. Amer Radio Relay League, 2017. ISBN: 9781625950727 
[7] GAELENS, J., VAN TORRE, P., VERHAEVERTET, J., al. LoRa mobile-to-base-station channel characterization in the Antarctic. Sensors, 2017, vol. 17 , no. 8, p. 1-18. DOI: $10.3390 /$ s 17081903

[8] JOVALEKIC, N., DRNDAREVIC, V., PIETROSEMOLI, E., et al Experimental study of LoRa transmission over seawater. Sensors 2018, vol. 18 , no. 9 , p. 1-23. DOI: $10.3390 / \mathrm{s} 18092853$

[9] ITU-T Recommendation V23. 600/1200-Baud Modem Standardized for Use in the General Switched 670 Telephone Network. 1988, 1993

[10] RICE, P.L., LONGLEY, A.G., NORTON, K. A., et al. Transmission Loss Predictions for Tropospheric Communications Circuits. Technical Note 101, revised 1/1/1967, U.S. Department of Commerce NBS-NIST.

[11] POSHALA, P., RUSHIL, K.K., GUPTA, R. Signal Chain Noise Figure Analysis. Application Report SLAA652, Texas Instruments, 2014.

[12] SANTILli, G., VENDITTOZZI, C, CAPPELlETTI, C., et al. CubeSat constellations for disaster management in remote areas. Acta Astronautica, 2018, vol. 145, p. 11-17. DOI: $10.1016 /$ j.actaastro.2017.12.050

[13] BAUER, F. H., TAYLOR, D., WHITE, R. A., et al. Educational outreach and international collaboration through ARISS: Amateur radio on the International Space Station. Chapter in Space Operations: Inspiring Humankind's Future. Springer, 2019. ISBN: 9783030115364 DOI: 10.1007/978-3-030-11536-4_33

\section{About the Authors...}

Manuel PRIETO was born in Madrid in 1974. He is currently Associate Professor in the University of Alcalá, Spain. He obtained his first degree in Telecommunications Engineering in 1995. In 1999, he obtained his degree in Electronics Engineering. Finally, he obtained his $\mathrm{PhD}$ by the University of Alcalá in 2005. His research interests are FPGA based digital electronics and radio-communications. He has taken part in multiple research projects in the field of hardware and software development for space. Since 2015, Dr. Prieto is involved in two research projects in the Antarctica for the automation of measurement.

Miguel Ángel DE PABLO has a BSc and a MSc degree in Geology (2002). He received two $\mathrm{PhD}$ degrees from Rey Juan Carlos Uiversity (2009) and Complutense University (2015) with two PhD Thesis about Mars Geology based on remote sensing. He is teaching at the Faculty of Sciences of the Alcalá Univesity since 2006, as Associate Professor since 2018. His research interest includes permafrost thermal monitoring, glacial and periglacial geomorphology and mapping of planetary surfaces (Earth and Mars). He has been working about Antarctic Permafrost since 2008.

Miguel RAMOS is currently Professor at the Department of Physics and Mathematics in the University of Alcalá (Spain). He concluded his degree in Fundamental Physics in 1980, earned his MSc in 1981 and his $\mathrm{PhD}$ on 1984. His research activities are currently developed through the University of Alcalá and the Spanish polar program. His main scientific interests are currently related to the characterization polar permafrost. Presently, he is co-I in the REMS project that is and instrument included in the Mars Science laboratory NASA mission and is co-I in the Mars 2020 project of NASA too.

Juan Javier JIMÉNEZ is Technical Engineer of Telecommunication (1980) by the Polytechnic University of Madrid, European Engineer by Feani (1995), Graduated in Environmental Sciences (2013) by the University of Alcalá. PDD by IESE Business School (2001). International Executive Coaching by LaSalle International Graduate School (2010). Master in Geographic Information Technologies (2014) by the University of Alcalá. Doctorate (since 2014) in the Space Research and Astrobiology Program at the University of Alcalá. Associate Researcher at the Geographical Studies Center/IGOT of the University of Lisbon and Researcher in PermaSnow Project. 